\title{
Relaxation Oscillations and Dynamical Properties in Two Time-Delay Slow-Fast Modified Leslie-Gower Models
}

\author{
Yufeng Wang, Youhua Qian $(\mathbb{D}$, and Bingwen Lin \\ College of Mathematics and Computer Science, Zhejiang Normal University, Jinhua 321004, China \\ Correspondence should be addressed to Youhua Qian; qyh2004@zjnu.cn
}

Received 13 July 2020; Revised 29 August 2020; Accepted 15 September 2020; Published 25 September 2020

Academic Editor: Yongjian Liu

Copyright (c) 2020 Yufeng Wang et al. This is an open access article distributed under the Creative Commons Attribution License, which permits unrestricted use, distribution, and reproduction in any medium, provided the original work is properly cited.

In this paper, we consider two kinds of time-delay slow-fast modified Leslie-Gower models. For the first system, we prove the existence and uniqueness of relaxation oscillation cycle through the geometric singular perturbation theory and entry-exit function. For the second system, we put forward a conjecture that the relaxation oscillation of the system is unique. Numerical simulation also verifies our results for the systems.

\section{Introduction}

In 2003, Alaoui and Okiye proposed the next modified Leslie-Gower model:

$$
\begin{aligned}
& \frac{\mathrm{d} x}{\mathrm{~d} T}=\left(r_{1}-b_{1} x-\frac{a_{1} y}{x+k_{1}}\right) x, \\
& \frac{\mathrm{d} y}{\mathrm{~d} T}=\left(r_{2}-\frac{a_{2} y}{x+k_{2}}\right) y,
\end{aligned}
$$

where $x$ is the density of a prey, $y$ is the density of a predator, and the parameters $r_{1}, k_{1}, a_{1}, b_{1}, r_{2}, k_{2}$, and $a_{2}$ are positive, which play biology roles.

Consider the rescaling,

$$
\begin{aligned}
x & =\frac{r_{1}}{b_{1}} \bar{x}, \\
y & =\frac{r_{1} r_{2}}{a_{2} b_{1}} \bar{y}, \\
T & =\frac{1}{r_{1}} t .
\end{aligned}
$$

Substitute (2) into (1), and we have $\bar{x}$ and $\bar{y}$ in terms of $x$ and $y$, then we have

$$
\begin{aligned}
& \frac{\mathrm{d} x}{\mathrm{~d} t}=x^{\prime}=x(1-x)-\frac{a x y}{x+e_{1}}, \\
& \frac{\mathrm{d} y}{\mathrm{~d} t}=y^{\prime}=\varepsilon y\left(1-\frac{y}{x+e_{2}}\right),
\end{aligned}
$$

where $a=\left(a_{1} r_{2} / a_{2} r_{1}\right), e_{1}=\left(b_{1} k_{1} / r_{1}\right), e_{2}=\left(b_{1} k_{2} / r_{1}\right)$, and $\varepsilon=\left(r_{2} / r_{1}\right)$. The parameter $\varepsilon$ can be regard as a small parameter in some special models, such as prey hares and predator lynx.

Consider the case where $\varepsilon$ is small enough, system (3) could be treat as a slow-fast system which has the fast variable $x$ and the slow one $y$. Applying the geometric singular perturbation theory, we could study the slow-fast systems $[4,5]$.

The geometric singular perturbation theory is mathematical rigorous that can analyze dynamics of some slowfast systems. The study of invariant manifolds in Fenichel's theory [3] is the basis of geometric singular perturbation theory, and the theory guarantees that any compact normally hyperbolic submanifold So of the critical manifold Co could perturb a locally invariant manifold $S_{\varepsilon}(0<\varepsilon \ll 1)$, which is $O(\varepsilon)$, close to $S o$. In recent years, geometric singular perturbation theory contains a fairly broad class of geometric points used to study the slow-fast systems, see, for example, [6-12]. And, for the exchange lemma, see [13, 14]. 
Recently, Wang and Zhang proved the existence and uniqueness of relaxation oscillation cycle of the slow-fast modified Leslie-Gower model [15]. Valery et al. studied the global dynamics in the Leslie-Gower model with the Allee Effect [16]. Du et al. considered two delays induced Hopf bifurcation and double Hopf bifurcation in a diffusive LeslieGower predator-prey system [17]. Karl et al. discussed the bifurcation of critical sets and relaxation oscillations in singular fast-slow systems [18]. Wang and Zhang considered the stability loss delay and smoothness of the return map in slow-fast systems [19]. Ambrosio et al. addressed the canard phenomenon in a slow-fast modified Leslie-Gower model [20]. Xia et al. discussed relaxation oscillations and the mechanism in a periodically excited vector field with pitchfork-Hopf bifurcation [21]. Atabaigi and Barati studied relaxation oscillations and canard explosion in a predatorprey system of Holling and Leslie types [22]. Ai and Sadhu considered the entry-exit theorem and relaxation oscillations in slow-fast planar systems [23].

The rest of the paper is organized as follows: Section 2 introduces the entry-exit function theory. Section 3 studies the first time-delay slow-fast modified Leslie-Gower model. Section 4 investigates another kind of time-delay slow-fast modified Leslie-Gower model.

\section{Entry-Exit Function}

We are going to consider a slow-fast vector field in the form of

$$
\begin{aligned}
& \frac{\mathrm{d} x}{\mathrm{~d} t}=f(x, y, \varepsilon) x, \\
& \frac{\mathrm{d} y}{\mathrm{~d} t}=\varepsilon g(x, y, \varepsilon),
\end{aligned}
$$

where $(x, y) \in \mathbb{R}^{2}$ are state-space variables and the parameter $\varepsilon$ represents the ratio of time scales. We define the functions $f$ and $g$ satisfying

$$
\begin{aligned}
& f(0, y, 0)<0, \quad \text { for } y>0, \\
& f(0, y, 0)>0, \quad \text { for } y<0, \\
& g(0, y, 0)<0 .
\end{aligned}
$$

For system (4), the $y$-axis consists of equilibria when $\varepsilon=$ 0 and $Y^{+}:=\{(x, y) \mid x=0, y>0\}$ attracting and $Y^{-}:=\{(x$, $y) \mid x=0, y<0\}$ repelling (Figure 1(a)). The Figure 1(b) is the case for $\varepsilon>0$, since the orbit of system (4) is attracted by the $y$-axis, the orbit starting at $\left(x_{0}, y_{0}\right)$ with $y_{0}>0$ and $x_{0}>0$ slightly moves toward the downside at the speed of $\varepsilon$. After the orbit passes positive $x$-axis, the $y$-axis begins repelling, then the orbit tends to move away from the negative $y$-axis at the point $\left(0, p_{0}\left(y_{0}\right)\right)$, and then it intersects the line $x=x_{0}$ at the point whose $y$-coordinate saying $p_{\varepsilon}\left(y_{0}\right)$ satisfies $\lim _{\varepsilon \longrightarrow 0} p_{\varepsilon}\left(y_{0}\right)=p_{0}\left(y_{0}\right)$, where $p_{0}\left(y_{0}\right)$ is determined by

$$
\int_{y_{0}}^{p_{0}\left(y_{0}\right)} \frac{f(0, y, 0)}{g(0, y, 0)} \mathrm{d} y=0 .
$$

\section{System with Two Positive Time Delays}

Consider a system with time delays $\tau_{1}$ and $\tau_{2}$, which are sufficiently small constants:

$$
\begin{aligned}
& \frac{\mathrm{d} x}{\mathrm{~d} t}=x^{\prime}=x(1-x)-\frac{a y}{x+e_{1}} x\left(t-\tau_{1}\right), \\
& \frac{\mathrm{d} y}{\mathrm{~d} t}=y^{\prime}=\varepsilon y\left(1-\frac{y\left(t-\tau_{2}\right)}{x+e_{2}}\right) .
\end{aligned}
$$

Using the Taylor formula,

$$
\begin{aligned}
& x\left(t-\tau_{1}\right) \approx x(t)-x^{\prime}(t) \tau_{1}, \\
& y\left(t-\tau_{2}\right) \approx y(t)-y^{\prime}(t) \tau_{2} .
\end{aligned}
$$

Substitute (8) into system (7), it yields

$$
\begin{aligned}
\frac{\mathrm{d} x}{\mathrm{~d} t} & =x^{\prime}=\left(x(1-x)-\frac{a x y}{x+e_{1}}\right) \frac{x+e_{1}}{x+e_{1}-a y \tau_{1}} \\
& :=x f(x, y, \varepsilon), \\
\frac{\mathrm{d} y}{\mathrm{~d} t} & =y^{\prime}=\varepsilon y\left(1-\frac{y}{x+e_{2}}\right) \frac{x+e_{2}}{x+e_{2}-\varepsilon y \tau_{2}}:=\varepsilon g(x, y, \varepsilon) .
\end{aligned}
$$

\subsection{Equilibria}

Lemma 1. For system (9), we have the following result: system (9) and system (3) have the same equilibria. So, that system (9) has equilibria $E_{1}(0,0), E_{2}\left(0, e_{2}\right)$, and $E_{3}(1,0)$.

Moreover, if $a e_{2}-e_{1}<0$, system (9) has a unique positive equilibrium $E_{*}\left(x_{*}, y_{*}\right)$ with $x_{*}=\left(\left(-\left(a+e_{1}-1\right)+\right.\right.$ $\left.\left.\sqrt{\left(a+e_{1}-1\right)^{2}-4\left(a e_{2}-e_{1}\right)}\right) / 2\right), y_{*}=x_{*}+e_{2}$.

3.2. Hopf Bifurcation. We consider the bifurcation of system (9) at the unique positive equilibrium $E_{*}\left(x_{*}, y_{*}\right)$ about parameter $\tau_{1}$. For convenience, we denote

$$
\begin{aligned}
& F(x, y)=x\left((1-x)-\frac{a x y}{x+e_{1}}\right) \frac{x+e_{1}}{x+e_{1}-a y \tau_{1}}, \\
& G(x, y)=\varepsilon y\left(1-\frac{y}{x+e_{2}}\right) \frac{x+e_{2}}{x+e_{2}-\varepsilon y \tau_{2}},
\end{aligned}
$$

so the linearized system of system (9) at the unique positive equilibrium $E_{*}\left(x_{*}, y_{*}\right)$ could be written as

$$
\left(\begin{array}{c}
x^{\prime} \\
y^{\prime}
\end{array}\right)=\left(\begin{array}{ll}
F_{x} & F_{y} \\
G_{x} & G_{y}
\end{array}\right)\left(\begin{array}{l}
x \\
y
\end{array}\right),
$$

with 




(a)

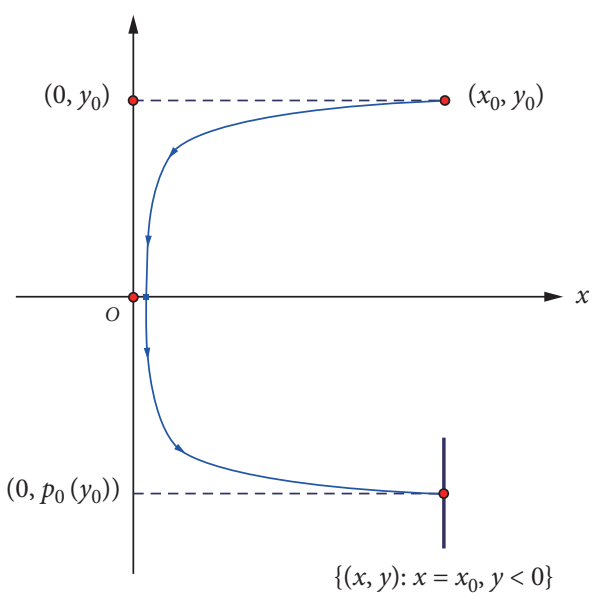

(b)

FIGURE 1: (a) For $\varepsilon=0$, the orbits of system (4) is shown and the $y$-axis consists of equilibria. (b) For $0<\varepsilon \ll 1$, a symbolic orbit of system (4) starts at $\left(x_{0}, y_{0}\right)$ and ends at $\left\{x=x_{0}, y_{0}<0\right\}$.

$$
\begin{aligned}
& F_{x}=\frac{-2 x_{*}^{2}-\left(e_{1}-1\right) x_{*}}{x_{*}+e_{1}-a y_{*} \tau_{1}}, \\
& F_{y}=\frac{-a x_{*}}{x_{*}+e_{1}-a y_{*} \tau_{1}}, \\
& G_{x}=\frac{\varepsilon\left(x_{*}+e_{2}\right)}{x_{*}+e_{2}-\varepsilon y_{*} \tau_{2}}, \\
& G_{y}=\frac{\varepsilon-2}{1-\varepsilon \tau_{2}} .
\end{aligned}
$$

Then, the characteristic equation of system (11) is

$$
\lambda^{2}-\left(F_{x}+G_{y}\right) \lambda-G_{x} F_{y}=0 \text {. }
$$

Lemma 2. For equation (13), we have the following result. If $\tau_{1}=\left(1-\varepsilon \tau_{2} /\left((2-\varepsilon) a e_{2}\right)\right)\left(1+\left(2-\varepsilon /\left(1-\varepsilon \tau_{2}\right)\right)(1-a)-e_{1}\right.$ $-2 a) x_{*}+\left(e_{1} / a e_{2}\right)$, then equation (13) has a pair of pure imaginary roots.

Proof. The condition of equation (13) has a pair of pure imaginary roots:

$$
F_{x}+G_{y}=0, \quad G_{x} F_{y}<0 .
$$

Consider $\varepsilon, \tau_{1}$, and $\tau_{2}$ are sufficiently small constants. We can easily get $G_{x}>0, F_{y}<0$, so $G_{x} F_{y}<0$ holds.

According to the equation $F_{x}+G_{y}=0$, we reach

$$
\frac{-2 x_{*}^{2}-\left(e_{1}-1\right) x_{*}}{x_{*}+e_{1}-a y_{*} \tau_{1}}+\frac{\varepsilon-2}{1-\varepsilon \tau_{2}}=0 \text {. }
$$

Taking $y_{*}=x_{*}+e_{2}$ into equation (15), then we get

$$
\frac{2 x_{*}^{2}+\left(e_{1}-1\right) x_{*}}{x_{*}+e_{1}-a\left(x_{*}+e_{2}\right) \tau_{1}}=\frac{\varepsilon-2}{1-\varepsilon \tau_{2}},
$$

i.e.,

$$
\tau_{1}=\frac{1-\varepsilon \tau_{2}}{(2-\varepsilon) a e_{2}}\left(1+\frac{2-\varepsilon}{1-\varepsilon \tau_{2}}(1-a)-e_{1}-2 a\right) x_{*}+\frac{e_{1}}{a e_{2}}
$$

The proof is completed.

Let $\lambda(\tau)=p(\tau) \pm i q(\tau)$ be the pair of pure imaginary roots of equation (13) that are satisfying $p\left(\tau_{1}\right)=0, q\left(\tau_{1}\right) \neq 0$. Then the following transversality condition holds.

Lemma 3. If $a \neq \sqrt{\left(a+e_{1}-1\right)^{2}-4\left(a e_{2}-e_{1}\right)}$ holds, then $\lambda^{\prime}\left(\tau_{1}\right) \neq 0$.

Proof. The condition of equation (13) has a pair of pure imaginary roots:

$$
\begin{aligned}
\operatorname{Re}\left(\frac{\mathrm{d} \lambda}{\mathrm{d} \tau_{1}}\right)= & \frac{F_{x}+G_{y}}{2 a}=\frac{1}{2 a} \frac{\mathrm{d} F_{x}}{\mathrm{~d} \tau_{1}}=\frac{x_{*}}{\left(x_{*}+e_{1}-a y_{*} \tau_{1}\right)^{2}} \\
& \cdot\left(2 x_{*}+e_{1}-1\right)=\frac{x_{*}}{\left(x_{*}+e_{1}-a y_{*} \tau_{1}\right)^{2}} \\
& \cdot\left(-a+\sqrt{\left(a+e_{1}-1\right)^{2}-4\left(a e_{2}-e_{1}\right)}\right) \neq 0 .
\end{aligned}
$$

The proof is completed.

Denote $\tau_{1}^{\prime}=\left(\left(1-\varepsilon \tau_{2}\right) /\left((2-\varepsilon) a e_{2}\right)\right)\left(1+\left(2-\varepsilon /\left(1-\varepsilon \tau_{2}\right)\right)\right.$ $\left.(1-a)-e_{1}-2 a\right) x_{*}+\left(e_{1} / a e_{2}\right)$. Combining Lemma 2 and Lemma 3, we conclude the following.

Theorem 1. For system (9), if $\tau_{1}=\tau_{1}^{\prime}$ and $a \neq$ $\sqrt{\left(a+e_{1}-1\right)^{2}-4\left(a e_{2}-e_{1}\right)}$, then the equilibrium $E_{*}\left(x_{*}\right.$, $\left.y_{*}\right)$ is a Hopf bifurcation point and a limit cycle occurs.

3.3. Dynamics of Limit Systems. Consider the limit systems of system (9). Setting $\varepsilon=0$ in (9), we reach the fast subsystem: 


$$
\begin{aligned}
& \frac{\mathrm{d} x}{\mathrm{~d} t}=x^{\prime}=\left(x(1-x)-\frac{a x y}{x+e_{1}}\right) \frac{x+e_{1}}{x+e_{1}-a y \tau_{1}}, \\
& \frac{\mathrm{d} y}{\mathrm{~d} t}=y^{\prime}=0 .
\end{aligned}
$$

Consider the slow time scale $\tau=\varepsilon t$ and take the singular again, we get the slow subsystem:

$$
\begin{aligned}
0 & =\left(x(1-x)-\frac{a x y}{x+e_{1}}\right) \frac{x+e_{1}}{x+e_{1}-a y \tau_{1}}, \\
\frac{\mathrm{d} y}{\mathrm{~d} \tau} & =\dot{y}=y\left(1-\frac{y}{x+e_{2}}\right) \frac{x+e_{2}}{x+e_{2}-\varepsilon y \tau_{2}},
\end{aligned}
$$

which is a differential-algebraic equation on the critical set:

$$
C_{0}=\left\{(x, y) \mid x=0 \text { or } y=\frac{(1-x)\left(x+e_{1}\right)}{a}:=F(x)\right\} \text {. }
$$

We restrict the parameters $a, e_{1}$, and $e_{2}$ to a subset

$$
U=\left\{\left(a, e_{1}, e_{2}\right) \mid \sqrt{\left(a+e_{1}-1\right)^{2}-4\left(a e_{2}-e_{1}\right)}<a<\frac{e_{1}}{e_{2}}\right\} .
$$

Under this restriction, $E_{*}\left(x_{*}, y_{*}\right)$ and $E_{2}\left(0, e_{2}\right)$ are located in the exclusion part of $C_{0}$. Some studies have shown that the branch $\{(x, y) \mid y=F(x), x>0, y>0\}$ of $C_{0}$ has a unique generic fold point $D\left(x_{M}, y_{M}\right)=\left(\left(1-e_{1} / 2\right), F(1\right.$ $\left.\left.-e_{1} / 2\right)\right)$. And, the two branches of $C_{0}$ cross transversally at the transcritical point $E(x, y)=\left(0,\left(e_{1} / a\right)\right)$ (Figure 2$)$. Then, the critical set $C_{0}$ is divided into four parts by points $D$ and E:

$$
\begin{aligned}
C_{0}^{a} & =\left\{(x, y) \mid \frac{\left(1-e_{1}\right)}{2}<x<1, y=F(x)\right\}, C_{0}^{+} \\
& =\left\{(x, y) \mid x=0, y>\frac{e_{1}}{a}\right\}, \\
C_{0}^{r} & =\left\{(x, y) \mid 0<x<\frac{\left(1-e_{1}\right)}{2}, y=F(x)\right\}, C_{0}^{-} \\
& =\left\{(x, y) \mid x=0,0<y<\frac{e_{1}}{a}\right\},
\end{aligned}
$$

where $C_{0}^{r}$ and $C_{0}^{-}$are normally hyperbolic repelling and $C_{0}^{a}$ and $C_{0}^{+}$are normally hyperbolic attracting.

In fact, when the parameter is limited to $U, E_{*}\left(x_{*}, y_{*}\right)$ is on $C_{0}^{r}$ and $E_{2}\left(0, e_{2}\right)$ is on $C_{0}^{-}$.

\subsection{Relaxation Oscillation}

Lemma 4. For system (9), there exists a unique $\bar{y}^{*}\left(e_{2}<\bar{y}^{*}<\left(e_{1} / a\right)\right)$ such that,

$$
\int_{\bar{y}^{*}}^{y_{M}} \frac{f(0, y, 0)}{g(0, y, 0)} \mathrm{d} y=0
$$

Proof. Considering the system (9), for $\bar{y} \in\left(e_{2},\left(e_{1} / a\right)\right)$,

$$
I(\bar{y})=\int_{\bar{y}}^{y_{M}} \frac{f(0, y, 0)}{g(0, y, 0)} \mathrm{d} y=\int_{\bar{y}}^{y_{M}} \frac{1-\left(a y / e_{1}\right)}{y\left(1-\left(y / e_{2}\right)\right)} \cdot \frac{e_{1}}{e_{1}-a y \tau_{1}} \mathrm{~d} y .
$$

So that

$$
I(\bar{y}) \longrightarrow-\infty \text { as } \bar{y} \longrightarrow e_{2}^{+}
$$

Furthermore

$$
\begin{aligned}
\mathrm{I}\left(\frac{e_{1}}{a}\right) & =\int_{\left(e_{1} / a\right)}^{y_{M}} \frac{f(0, y, 0)}{g(0, y, 0)} \mathrm{d} y \\
& =\int_{\left(e_{1} / a\right)}^{y_{M}} \frac{1-\left(a y / e_{1}\right)}{y\left(1-\left(y / e_{2}\right)\right)} \cdot \frac{e_{1}}{e_{1}-a y \tau_{1}} \mathrm{~d} y>0 .
\end{aligned}
$$

Then, we combine (25) and (27) and conclude that there exist a unique $\bar{y}^{*}\left(e_{2}<\bar{y}^{*}<\left(e_{1} / a\right)\right)$ such that,

$$
\int_{\bar{y}^{*}}^{y_{M}} \frac{f(0, y, 0)}{g(0, y, 0)} \mathrm{d} y=0
$$

The proof is completed.

Let us define $x_{r}$ to be the $x$-coordinate of the intersection point of $y=\bar{y}^{*}$ and $C_{0}^{a}$. Then, define a singular slow-fast cycle $\gamma_{0}$, which contains two slow segments on $C_{0}^{a}$ from $\left(x_{r}, \bar{y}^{*}\right)$ to $\left(x_{M}, y_{M}\right)$ and on the positive $y$-axis from $\left(0, y_{M}\right)$ to $\left(0, \bar{y}^{*}\right)$ and the two fast connections from $\left(x_{M}, y_{M}\right)$ to $\left(0, y_{M}\right)$ and $\left(0, \bar{y}^{*}\right)$ to $\left(x_{r}, \bar{y}^{*}\right)$, respectively. The next theorem explains the existence and uniqueness of the relaxation oscillation.

Theorem 2. For system (9), restrict the parameters to subset $U$ and let $V$ be a tubular neighborhood of $\gamma_{0}$. Then, for each fixed $\varepsilon>0$ sufficiently small, system (9) has a unique limit cycle $\gamma_{\varepsilon} \subset V$, which is strongly attracting. Moreover, the cycle $\gamma_{\varepsilon}$ is the unique limit cycle that converges to $\gamma_{0}$ in the Hausdorff distance [3] as $\varepsilon \longrightarrow 0$.

Proof. By Fenichel's theory, the critical submanifold $C_{0}^{a}$ perturbs to a nearby slow manifold $C_{\varepsilon}^{a}$, which is $O(\varepsilon)$ near $C_{0}^{a}$. By Theorem 2.1 of [9] on the analysis of a jump point, the slow manifold $C_{\varepsilon}^{a}$ can be continued and passes the fold point $D\left(x_{M}, y_{M}\right)$, and then it jumps to another attracting branch $C_{0}^{+}$.

Let $x_{l}$ be a sufficiently small positive number. Define two vertical sections $\Delta^{\text {in }}$ and $\Delta^{\text {out }}$ as shown in Figure 2:

$$
\Delta^{\text {in }}:=\left\{\left(x_{l}, y\right) \in V \mid y \in I_{\text {in }}\right\}, \quad \Delta^{\text {out }}:=\left\{\left(x_{l}, y\right) \in V \mid y \in I_{\text {out }}\right\},
$$

where $I_{\text {in }}$ and $I_{\text {out }}$ are closed intervals centered at $y_{M}$ and $y_{\text {out }}=p_{0}\left(y_{M}\right)$, respectively. Through the flow of system (4), we define the transition map $\Pi: \Delta^{\text {in }} \longrightarrow \Delta^{\text {in }}$, which is a composition of the next two maps:

$$
\Pi_{1}: \Delta^{\text {in }} \longrightarrow \Delta^{\text {out }}, \Pi_{2}: \Delta^{\text {out }} \longrightarrow \Delta^{\text {in }} .
$$

Then, $\Pi: \Delta^{\text {in }} \longrightarrow \Delta^{\text {in }}$ is given by the composition $\Pi:=\Pi_{2} \circ \Pi_{1}$. 


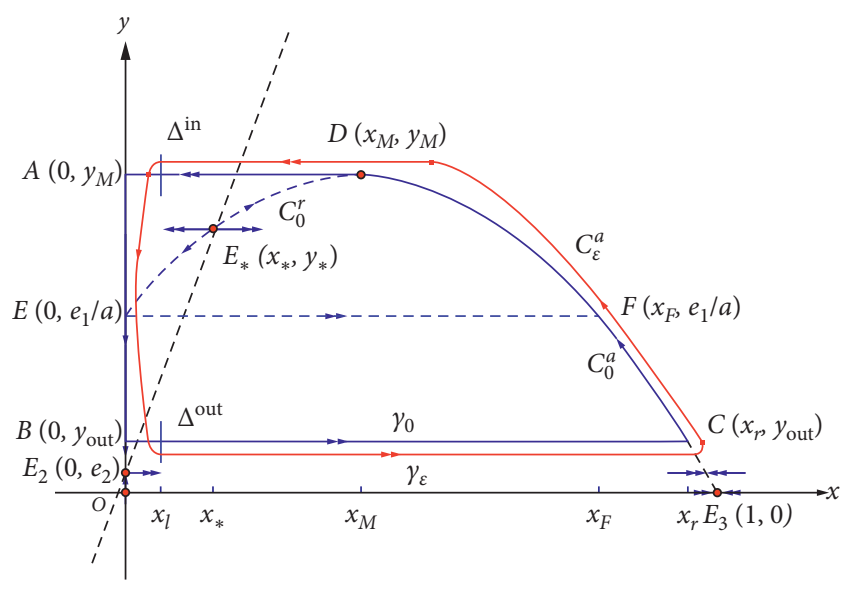

FIGURE 2: Illustration for the relaxation oscillations of system (9). The critical manifold $C_{0}$ contains repelling part $C_{0}^{r}$ and attracting part $C_{0}^{a}$. The dashed black line is the predator isocline, the red dot is the fold point $D\left(x_{M}, y_{M}\right)$, the solid blue line is the slow-fast cycle $\gamma_{0}$, the double and single arrows are fast and slow flows, and the solid red is the attracting relaxation oscillation $\gamma_{\varepsilon}$. $\Pi_{2}$

Now, we analyze the properties of these two maps $\Pi_{1}$ and

(a) Analysis of $\Pi_{1}$. Use the same proof as Lemma 4, for each $y_{0} \in I_{i n}$, we can define $p_{0}\left(y_{0}\right)$, with $e_{2}<p_{0}\left(y_{0}\right)<\left(e_{1} / a\right)$ through the following formula:

$$
\int_{p_{0}\left(y_{0}\right)}^{y_{0}} \frac{f(0, y, 0)}{g(0, y, 0)} \mathrm{d} y=0
$$

(b) Analysis of $\Pi_{2}$. Consider two orbits $\gamma_{\varepsilon}^{1}$ and $\gamma_{\varepsilon}^{2}$ starting on $\Delta^{\text {out }}$, from Fenichel's theory, $\gamma_{\varepsilon}^{1}$ and $\gamma_{\varepsilon}^{2}$ will be attracted to $C_{\varepsilon}^{a}$ at the exponential rate $O\left(e^{-1 / \varepsilon}\right)$. By Theorem 2.1 of [9], $\gamma_{\varepsilon}^{1}$ and $\gamma_{\varepsilon}^{2}$ pass by the generic fold point $D\left(x_{M}, y_{M}\right)$ contracting exponentially toward each other. Then, they fly to $\Delta^{\text {in }}$.

Therefore, according to the results of (a) and (b), we obtain that the transition map $\Pi: \Delta^{\text {in }} \longrightarrow \Delta^{\text {in }}$ is a contraction at the exponential rate $O\left(e^{-1 / \varepsilon}\right)$. Then, it follows from the contraction mapping theorem that $\Pi$ has a unique fixed point in $\Delta^{\text {in }}$, which must be stable. This fixed point provides a unique relaxation oscillation cycle $\gamma_{\varepsilon} \subset V$ of system (9) passing $\Delta^{\text {in }}$ for each $0<\varepsilon \ll 1$.

According to Fenichel's theory and Theorem 2.1 of [9], we obtain that the relaxation oscillation cycle $\gamma_{\varepsilon}$ converges to the slow-fast cycle $\gamma_{0}$ as $\varepsilon \longrightarrow 0$ in the Hausdorff distance.

So, we can get that the relaxation oscillation cycle $\gamma_{\varepsilon}$ is a unique limit cycle of system (9) located in $V$ for each $0<\varepsilon \ll 1$.

The theorem is proved.
3.5. Numerical Simulation. First of all, we provide an example to illustrate Theorem 2.

Select the parameter values $\left(a, e_{1}, e_{2}, \varepsilon, \tau_{1}, \tau_{2}\right)=(1,0.2$, $0.022,0.01,0.2,0.2)$, and it is easy to state that the system (9) has a unique positive equilibrium point $E_{*}(0.33,0.36) . \mathrm{Nu}-$ merical simulation shows that it has a unique limit cycle. Figure 3 shows the phase portrait and the time series of the relaxation oscillation cycle.

(a) The orbit is shown in red in phase space

(b) Time series with the black dashed curve (prey) and red full curve (predator)

In addition, we compare the phase portrait and time series of system (9) with system (7). Figure 4 presents the phase portrait of system (9) and system (7). We can see that the two curves are very close to each other. Figure 5 shows the time series of system (9) and system (7).

(a) Time series for the relaxation oscillation of the predator with the red dashed curve (system (7)) and the blue full curve (system (9))

(b) Time series for the relaxation oscillation of the prey with the blue dashed curve (system (7)) and the red full curve (system (9)). Time series for the relaxation oscillation of prey with the blue dashed curve (system (4)) and the red full curve (system (6)).

\section{System with Two Time Delays}

In this section, we consider time delays $\tau_{1}$ and $\tau_{2}$ in system (3), then we have

$$
\begin{aligned}
& \frac{\mathrm{d} x}{\mathrm{~d} t}=x^{\prime}=x(1-x)-\frac{a x}{x+e_{1}} y\left(t-\tau_{1}\right), \\
& \frac{\mathrm{d} y}{\mathrm{~d} t}=y^{\prime}=\varepsilon y\left(1-\frac{y}{x\left(t-\tau_{2}\right)+e_{2}}\right),
\end{aligned}
$$

where $\tau_{1}$ stands for delayed maturation of the predator and $\tau_{2}$ stands for the time needed to digest the prey.

4.1. Equilibria and Hopf Bifurcation. System (32) has the same equilibria with system (9). So, system (32) has a unique positive equilibrium $E_{*}\left(x_{*}, y_{*}\right)$ with $x_{*}=\left(\left(-\left(a+e_{1}-1\right)+\right.\right.$ $\left.\left.\sqrt{\left(a+e_{1}-1\right)^{2}-4\left(a e_{2}-e_{1}\right)}\right) / 2\right), y_{*}=x_{*}+e_{2}$.

In order to get the conditions for Hopf bifurcation, we reach the linearized system of system (32) at the equilibrium $E_{*}\left(x_{*}, y_{*}\right)$ :

$$
\left(\begin{array}{l}
x^{\prime} \\
y^{\prime}
\end{array}\right)=A\left(\begin{array}{l}
x \\
y
\end{array}\right)+B\left(\begin{array}{c}
x\left(t-\tau_{1}\right) \\
y\left(t-\tau_{1}\right)
\end{array}\right)+C\left(\begin{array}{c}
x\left(t-\tau_{2}\right) \\
y\left(t-\tau_{2}\right)
\end{array}\right),
$$

where 


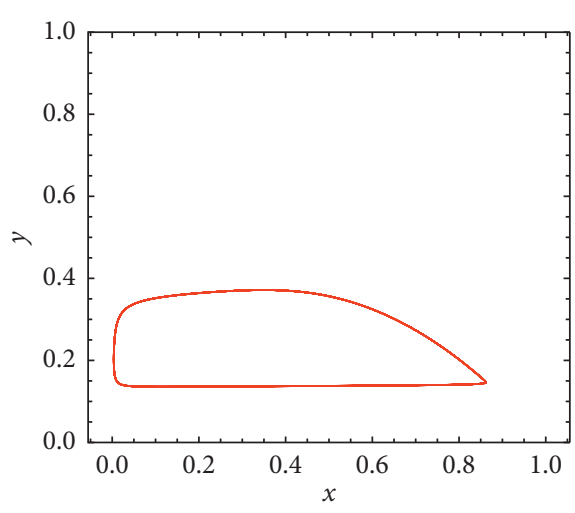

(a)

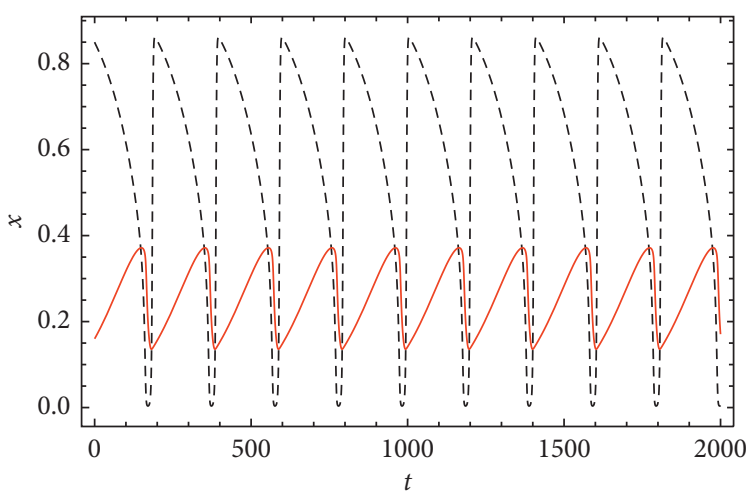

(b)

FIgURE 3: Numerical simulation of system (9).

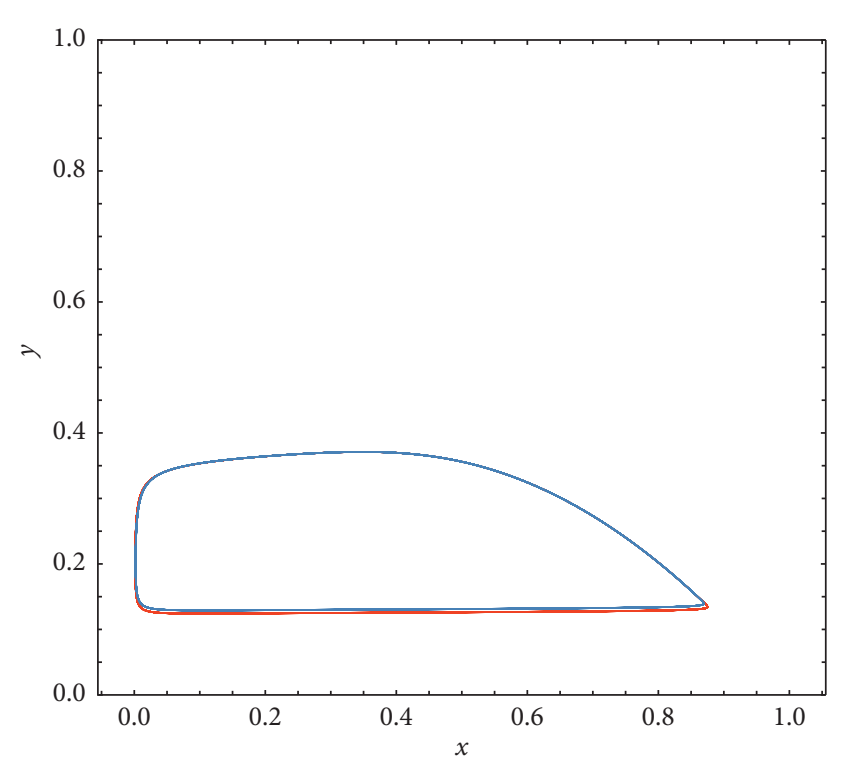

FIgURE 4: Phase space: the dashed blue curve shows the orbit of system (9) and the red full curve shows the orbit of system (7).

$$
\begin{aligned}
A & =\left(\begin{array}{cc}
1-2 x_{*}-\frac{a e_{1}}{\left(x+e_{1}\right)^{2}} y\left(t-\tau_{1}\right) & 0 \\
0 & \varepsilon\left(1-\frac{2 y_{*}}{x\left(t-\tau_{2}\right)+e_{2}}\right)
\end{array}\right), \\
B & =\left(\begin{array}{cc}
0 \frac{-a x_{*}}{x_{*}+e_{1}} \\
0
\end{array}\right), \\
C & =\left(\begin{array}{c}
0 \\
\frac{\varepsilon y_{*}^{2}}{\left(x\left(t-\tau_{2}\right)+e_{2}\right)^{2}}
\end{array}\right) .
\end{aligned}
$$

Substituting $y_{*}=x_{*}+e_{2}$ into (34) yields

$$
\begin{aligned}
A & =\left(\begin{array}{cc}
1-2 x_{*}-\frac{a e_{1}}{\left(x+e_{1}\right)^{2}} y\left(t-\tau_{1}\right) & 0 \\
0 & \varepsilon\left(1-\frac{2\left(x_{*}+e_{2}\right)}{x\left(t-\tau_{2}\right)+e_{2}}\right)
\end{array}\right), \\
B & =\left(\begin{array}{cc}
0 \frac{-a x_{*}}{x_{*}+e_{1}} \\
0
\end{array}\right), \\
C & =\left(\begin{array}{c}
0 \\
\frac{\varepsilon\left(x_{*}+e_{2}\right)^{2}}{0}
\end{array}\right),
\end{aligned}
$$

i.e.,

$$
\begin{aligned}
A & =\left(\begin{array}{cc}
\frac{x_{*}}{x_{*}+e_{1}}\left(2 x_{*}+e_{1}-1\right) & 0 \\
0 & -\varepsilon
\end{array}\right), \\
B & =\left(\begin{array}{cc}
0 & \frac{-a x_{*}}{x_{*}+e_{1}} \\
0 & 0
\end{array}\right), \\
C & =\left(\begin{array}{cc}
0 & 0 \\
\varepsilon & 0
\end{array}\right) .
\end{aligned}
$$

Denote $\tau=\tau_{1}+\tau_{2}$, the associated characteristic equation of system (33) can be obtained as follows:

$$
\begin{aligned}
\lambda^{2}- & \left(\frac{x_{*}}{x_{*}+e_{1}}\left(2 x_{*}+e_{1}-1\right)+\varepsilon\right) \lambda+\varepsilon \\
& \left(\frac{a x_{*}}{x_{*}+e_{1}} e^{-\lambda \tau}+\frac{x_{*}}{x_{*}+e_{1}}\left(2 x_{*}+e_{1}-1\right)\right)=0 .
\end{aligned}
$$




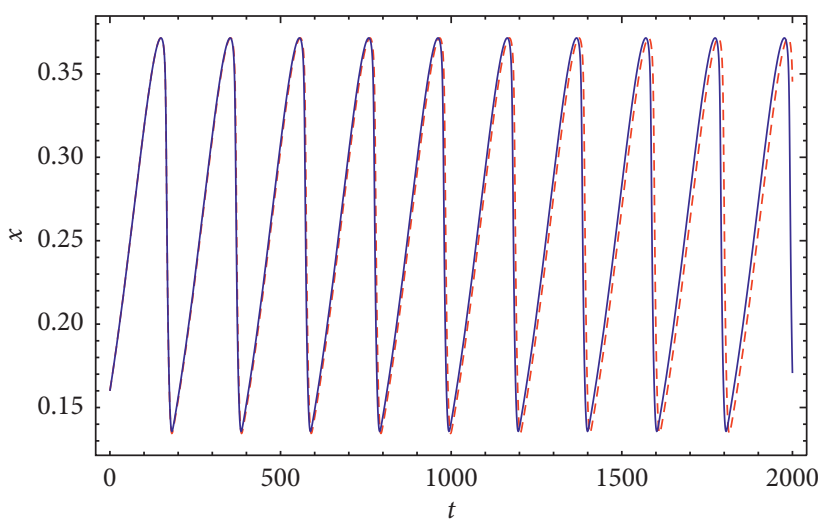

(a)

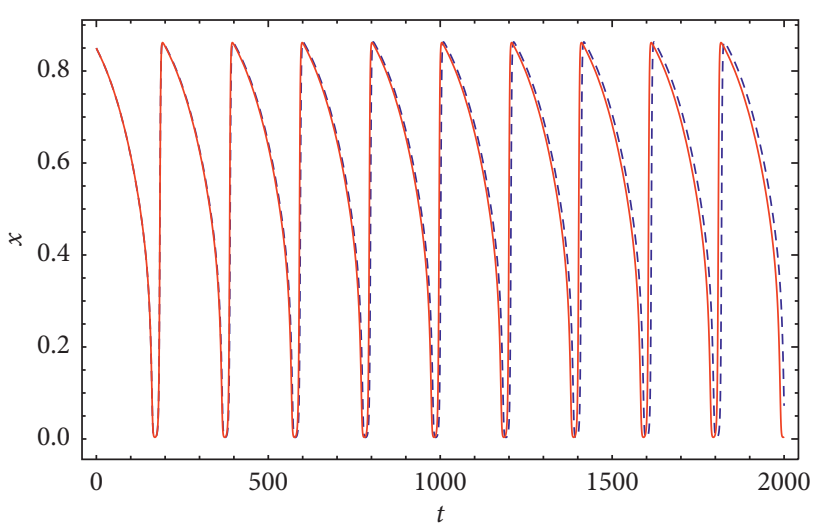

(b)

Figure 5: Time series of system (9) and system (7).

Lemma 5. For equation (37), if $\sqrt{\left(a+e_{1}-1\right)^{2}-4\left(a e_{2}-e_{1}\right)}$ $\geq 2 a$, then equation (37) ha no pairs of pure imaginary roots. If $\sqrt{\left(a+e_{1}-1\right)^{2}-4\left(a e_{2}-e_{1}\right)}<2 a$, then equation (37) has a pair of pure imaginary roots.

Proof. Let $\lambda= \pm \omega i$ be the pair of pure imaginary roots of equation (37), then we take $\lambda=\omega i$ into equation (37) with $e^{-\lambda \tau}=e^{-\omega i \tau}=\cos (\omega \tau)-i \sin (\omega \tau)$, it yields

$$
\begin{aligned}
& -\omega^{2}-\left(\frac{x_{*}}{x_{*}+e_{1}}\left(2 x_{*}+e_{1}-1\right)+\varepsilon\right) \omega i, \\
& +\varepsilon\left(\frac{a x_{*}}{x_{*}+e_{1}}(\cos (\omega \tau)-i \sin (\omega \tau))+\frac{x_{*}}{x_{*}+e_{1}}\left(2 x_{*}+e_{1}-1\right)\right)=0 .
\end{aligned}
$$

Separating the real and imaginary parts of equation (38), then we get

$$
\begin{aligned}
& -\omega^{2}+\frac{\varepsilon x_{*}}{x_{*}+e_{1}}\left(2 x_{*}+e_{1}-1\right)+\frac{a \varepsilon x_{*}}{x_{*}+e_{1}} \cos (\omega \tau)=0, \\
& \omega\left(\frac{x_{*}}{x_{*}+e_{1}}\left(2 x_{*}+e_{1}-1\right)+\varepsilon\right)-\frac{a \varepsilon x_{*}}{x_{*}+e_{1}} \sin (\omega \tau)=0 .
\end{aligned}
$$

So that

$$
\begin{aligned}
& \cos (\omega \tau)=\frac{\omega^{2}-\left(\varepsilon x_{*} / x_{*}+e_{1}\right)\left(2 x_{*}+e_{1}-1\right)}{a \varepsilon x_{*} /\left(x_{*}+e_{1}\right)} \triangleq Q_{0}, \\
& \sin (\omega \tau)=\frac{\omega\left(\left(x_{*} / x_{*}+e_{1}\right)\left(2 x_{*}+e_{1}-1\right)+\varepsilon\right)}{a \varepsilon x_{*} /\left(x_{*}+e_{1}\right)} .
\end{aligned}
$$

According to $\sin ^{2}(\omega \tau)+\cos ^{2}(\omega \tau)=1$, we have

$$
\omega^{4}+D \omega^{2}+E=0
$$

with

$$
\begin{aligned}
D= & \left(\frac{x_{*}}{x_{*}+e_{1}}\left(2 x_{*}+e_{1}-1\right)+\varepsilon\right)^{2}-\frac{2 \varepsilon x_{*}}{x_{*}+e_{1}}\left(2 x_{*}+e_{1}-1\right) \\
= & \left(\frac{x_{*}}{x_{*}+e_{1}}\left(2 x_{*}+e_{1}-1\right)\right)^{2}+\varepsilon^{2}>0, \\
E= & \left(\frac{\varepsilon x_{*}}{x_{*}+e_{1}}\left(2 x_{*}+e_{1}-1\right)\right)^{2}-\left(\frac{a \varepsilon x_{*}}{x_{*}+e_{1}}\right)^{2}=\left(\frac{\varepsilon x_{*}}{x_{*}+e_{1}}\right)^{2} \\
& \cdot\left(\left(2 x_{*}+e_{1}-1\right)^{2}-a^{2}\right), \\
= & \left(\frac{\varepsilon x_{*}}{x_{*}+e_{1}}\right)^{2}\left(2 x_{*}+e_{1}-1+a\right)\left(2 x_{*}+e_{1}-1-a\right) .
\end{aligned}
$$

Since

$$
\begin{aligned}
& 2 x_{*}+e_{1}-1+a=\sqrt{\left(a+e_{1}-1\right)^{2}-4\left(a e_{2}-e_{1}\right)}>0, \\
& 2 x_{*}+e_{1}-1-a=\sqrt{\left(a+e_{1}-1\right)^{2}-4\left(a e_{2}-e_{1}\right)}-2 a .
\end{aligned}
$$

We can conclude that if $\sqrt{\left(a+e_{1}-1\right)^{2}-4\left(a e_{2}-e_{1}\right)}$ $\geq 2 a$, that $E \geq 0$, which implies that Equation (41) has no positive roots, so equation (37) has no pairs of pure imaginary roots. Besides, if $\sqrt{\left(a+e_{1}-1\right)^{2}-4\left(a e_{2}-e_{1}\right)}<$ $2 a$, then $E<0$ and equation (41) has a positive root:

$$
\omega=\sqrt{\frac{-D+\sqrt{D^{2}-4 E}}{2}},
$$

which implies equation (37) has a pair of pure imaginary roots when

$$
\tau_{0 j}=\frac{\arccos \left(Q_{0}\right)+2 j \pi}{\omega}, \quad j=0,1,2, \ldots
$$

The proof is completed. 
Denote $\lambda(\tau)=p(\tau) \pm i q(\tau)$ be the pair of pure imaginary roots of equation (37) that are satisfying $p\left(\tau_{0 j}\right)=0$, $q\left(\tau_{0 j}\right)=\omega$.

Lemma

$$
\text { 6. (transversality condition) If }
$$
$\sqrt{\left(a+e_{1}-1\right)^{2}-4\left(a e_{2}-e_{1}\right)}<2 a$ holds, then $p^{\prime}\left(\tau_{0 j}\right)>0$, $j=0,1,2, \ldots$.

Proof. Differentiating both sides of equation (37) with respect to $\tau$, we reach

$2 \lambda \frac{\mathrm{d} \lambda}{\mathrm{d} \tau}+\left(\frac{x_{*}}{x_{*}+e_{1}}\left(2 x_{*}+e_{1}-1\right)+\varepsilon\right) \frac{\mathrm{d} \lambda}{\mathrm{d} \tau}-\lambda \varepsilon \frac{a x_{*}}{x_{*}+e_{1}} e^{-\lambda \tau}=0$,

then

$$
\left(\frac{\mathrm{d} \lambda}{\mathrm{d} \tau}\right)^{-1}=\frac{2 \lambda+\left(x_{*} / x_{*}+e_{1}\right)\left(2 x_{*}+e_{1}-1\right)+\varepsilon}{\lambda \varepsilon\left(a x_{*} / x_{*}+e_{1}\right) e^{-\lambda \tau}} .
$$

So

$$
\begin{aligned}
\operatorname{Re}\left(\frac{\mathrm{d} \lambda}{\mathrm{d} \tau}\right)_{\tau=\tau_{0 j}}^{-1} & =\operatorname{Re}\left[\frac{2 \lambda+\left(x_{*} / x_{*}+e_{1}\right)\left(2 x_{*}+e_{1}-1\right)+\varepsilon}{\lambda \varepsilon\left(a x_{*} / x_{*}+e_{1}\right) e^{-\lambda \tau}}\right]_{\tau=\tau_{0 j}} \\
& =\frac{2 \omega \cos \omega \tau+\left[\left(a x_{*} / x_{*}+e_{1}\right)\left(2 x_{*}+e_{1}-1\right)+\varepsilon\right] \sin \omega \tau}{\left(\omega \varepsilon a x_{*} / x_{*}+e_{1}\right)}, \\
& =\frac{2 \omega^{2}+D}{\left(\varepsilon a x_{*} / x_{*}+e_{1}\right)^{2}}>0 .
\end{aligned}
$$

To sum up, we get $p^{\prime}\left(\tau_{0 j}\right)>0$.

The proof is completed.

Theorem 3. For system (32), if $\tau_{1}+\tau_{2}=\tau_{0 j}, j=0,1,2, \ldots$, system (32) undergoes a Hopf bifurcation and $\tau_{0 j}$ are the Hopf bifurcation values.

Proof. If $\tau_{1}+\tau_{2}=\tau_{0 j}, j=0,1,2, \ldots$ then the equation (37) has a pair of purely imaginary roots which satisfy the transversality condition. Thus system (32) undergoes a Hopf bifurcation and $\tau_{0 j}$ are the Hopf bifurcation values.

The theorem is proved.

Remark 1. If $0<\tau_{1}+\tau_{2}<\tau_{00}$, then $E_{*}\left(x_{*}, y_{*}\right)$ is locally asymptotically stable and if $\tau_{1}+\tau_{2}>\tau_{00}$, then $E_{*}\left(x_{*}, y_{*}\right)$ is unstable.

4.2. Analysis of Limit Systems. In order to get rid of the delay $\tau_{1}$ and $\tau_{2}$, we denote $x=x_{1}, \quad x\left(t-\tau_{2}\right)=x_{2}, \quad y=y_{1}$ $y\left(t-\tau_{1}\right)=y_{2}$. Then, we get

$$
\begin{aligned}
& \frac{\mathrm{d} x_{1}}{\mathrm{~d} t}=x_{1}\left(1-x_{1}\right)-\frac{a x_{1}}{x_{1}+e_{1}} y_{2}, \\
& \frac{\mathrm{d} x_{2}}{\mathrm{~d} t}=x_{2}\left(1-x_{2}\right)-\frac{a x_{2}}{x_{2}+e_{1}} y\left(t-\tau_{1}-\tau_{2}\right), \\
& \frac{\mathrm{d} y_{1}}{\mathrm{~d} t}=\varepsilon y_{1}\left(1-\frac{y_{1}}{x_{2}+e_{2}}\right), \\
& \frac{\mathrm{d} y_{2}}{\mathrm{~d} t}=\varepsilon y_{2}\left(1-\frac{y_{2}}{x\left(t-\tau_{1}-\tau_{2}\right)+e_{2}}\right),
\end{aligned}
$$

i.e.,

$$
\begin{aligned}
& \frac{\mathrm{d} x_{1}}{\mathrm{~d} t}=x_{1}\left(1-x_{1}\right)-\frac{a x_{1}}{x_{1}+e_{1}} y_{2}, \\
& \frac{\mathrm{d} x_{2}}{\mathrm{~d} t}=x_{2}\left(1-x_{2}\right)-\frac{a x_{2}}{x_{2}+e_{1}} y_{1}(t-\tau), \\
& \frac{\mathrm{d} y_{1}}{\mathrm{~d} t}=\varepsilon y_{1}\left(1-\frac{y_{1}}{x_{2}+e_{2}}\right), \\
& \frac{\mathrm{d} y_{2}}{\mathrm{~d} t}=\varepsilon y_{2}\left(1-\frac{y_{2}}{x_{1}(t-\tau)+e_{2}}\right) .
\end{aligned}
$$

When $\tau=0$, system (50) goes to

$$
\begin{aligned}
& \frac{\mathrm{d} x_{1}}{\mathrm{~d} t}=x_{1}\left(1-x_{1}\right)-\frac{a x_{1}}{x_{1}+e_{1}} y_{2}, \\
& \frac{\mathrm{d} x_{2}}{\mathrm{~d} t}=x_{2}\left(1-x_{2}\right)-\frac{a x_{2}}{x_{2}+e_{1}} y_{1}, \\
& \frac{\mathrm{d} y_{1}}{\mathrm{~d} t}=\varepsilon y_{1}\left(1-\frac{y_{1}}{x_{2}+e_{2}}\right), \\
& \frac{\mathrm{d} y_{2}}{\mathrm{~d} t}=\varepsilon y_{2}\left(1-\frac{y_{2}}{x_{1}+e_{2}}\right) .
\end{aligned}
$$

Consider the case where $\varepsilon$ is small enough, system (51) could be viewed as a slow-fast system which has the fast variable $X=\left(\begin{array}{l}x_{1} \\ x_{2}\end{array}\right)$ and the slow variable $Y=\left(\begin{array}{l}y_{1} \\ y_{2}\end{array}\right)$. We have

$$
\begin{aligned}
f(X, Y, \varepsilon) & =\left(f_{1}, f_{2}\right) \\
& =\left(x_{1}\left(1-x_{1}\right)-\frac{a x_{1}}{x_{1}+e_{1}} y_{2}, x_{2}\left(1-x_{2}\right)-\frac{a x_{2}}{x_{2}+e_{1}} y_{1}\right) .
\end{aligned}
$$

Then, we arrive at the critical set:

$C_{0}=\left\{(x, y) \in \mathbb{R}^{2} \times \mathbb{R}^{2} \mid f(X, Y, 0)=0\right\}=C_{0}^{1} \cup C_{0}^{2} \cup C_{0}^{3} \cup C_{0}^{4}$. 
with

$$
\begin{aligned}
& C_{0}^{1}=\left\{x_{1}=0, x_{2}=0\right\}, \\
& C_{0}^{2}=\left\{x_{1}=0, y_{1}=\frac{1}{a}\left(1-x_{2}\right)\left(x_{2}+e_{1}\right)\right\}, \\
& C_{0}^{3}=\left\{y_{2}=\frac{1}{a}\left(1-x_{1}\right)\left(x_{1}+e_{1}\right), x_{2}=0\right\}, \\
& C_{0}^{4}=\left\{y_{2}=\frac{1}{a}\left(1-x_{1}\right)\left(x_{1}+e_{1}\right), y_{1}=\frac{1}{a}\left(1-x_{2}\right)\left(x_{2}+e_{1}\right)\right\} .
\end{aligned}
$$

Since

$$
D_{X} f=\left(\begin{array}{cc}
1-2 x_{1}-\frac{a e_{1} y_{2}}{\left(x_{1}+e_{1}\right)^{2}} & 0 \\
0 & 1-2 x_{2}-\frac{a e_{1} y_{1}}{\left(x_{2}+e_{1}\right)^{2}}
\end{array}\right),
$$

Then, the eigenvalues of matrix (55) are

$$
\begin{aligned}
& \mu_{1}=1-2 x_{1}-\frac{a e_{1} y_{2}}{\left(x_{1}+e_{1}\right)^{2}}, \\
& \mu_{2}=1-2 x_{2}-\frac{a e_{1} y_{1}}{\left(x_{2}+e_{1}\right)^{2}} .
\end{aligned}
$$

Thus, we reach the attractive part and the repulsive part of $C_{0}^{1}$ are, respectively,

$$
\begin{aligned}
& C_{0}^{1 a}=\left\{x_{1}=0, x_{2}=0, y_{1}>\frac{e_{1}}{a}, y_{2}>\frac{e_{1}}{a}\right\}, \\
& C_{0}^{1 r}=\left\{x_{1}=0, x_{2}=0, y_{1}<\frac{e_{1}}{a}, y_{2}<\frac{e_{1}}{a}\right\} .
\end{aligned}
$$

The attractive part and the repulsive part of $C_{0}^{2}$ are, respectively,

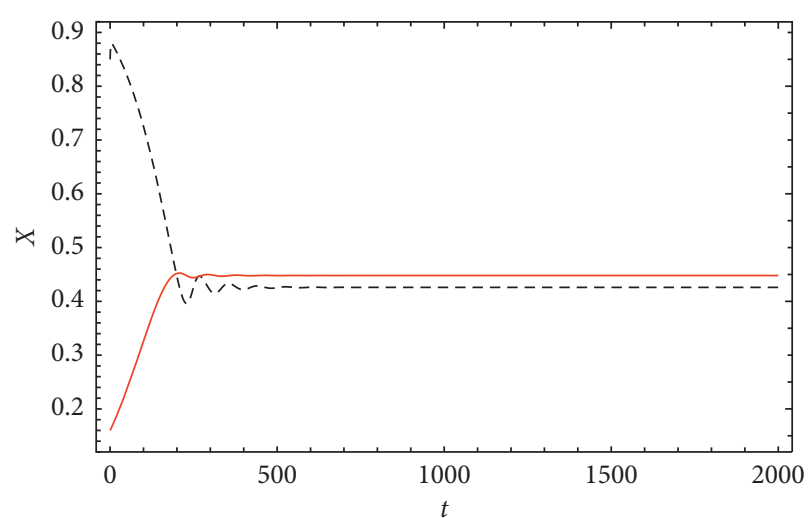

Figure 6: Choose $\left(\tau_{1}, \tau_{2}\right)=(3,1)$ satisfying $\tau_{1}+\tau_{2}=4<\tau_{00}$, then the equilibrium $E_{*}\left(x_{*}, y_{*}\right)$ of system (32) is asymptotically stable. Time series with the black dashed curve (prey) and red full curve (predator).

$$
\begin{aligned}
& C_{0}^{2 a}=\left\{x_{1}=0, x_{2}>\frac{1-e_{1}}{2}, y_{1}=\frac{1}{a}\left(1-x_{2}\right)\left(x_{2}+e_{1}\right), y_{2}>\frac{e_{1}}{a}\right\}, \\
& C_{0}^{2 r}=\left\{x_{1}=0,0<x_{2}<\frac{1-e_{1}}{2}, y_{1}=\frac{1}{a}\left(1-x_{2}\right)\left(x_{2}+e_{1}\right), y_{2}<\frac{e_{1}}{a}\right\}
\end{aligned}
$$

The attractive part and the repulsive part of $C_{0}^{3}$ are, respectively,

$$
\begin{aligned}
& C_{0}^{3 a}=\left\{x_{1}>\frac{1-e_{1}}{2}, x_{2}=0, y_{1}>\frac{e_{1}}{a}, y_{2}=\frac{1}{a}\left(1-x_{1}\right)\left(x_{1}+e_{1}\right)\right\}, \\
& C_{0}^{3 r}=\left\{0<x_{1}<\frac{1-e_{1}}{2}, x_{2}=0, y_{1}<\frac{e_{1}}{a}, y_{2}=\frac{1}{a}\left(1-x_{1}\right)\left(x_{1}+e_{1}\right)\right\} .
\end{aligned}
$$

and the attractive part and the repulsive part of $C_{0}^{4}$ are, respectively,

$$
\begin{aligned}
& C_{0}^{4 a}=\left\{x_{1}>\frac{1-e_{1}}{2}, x_{2}>\frac{1-e_{1}}{2}, y_{1}=\frac{1}{a}\left(1-x_{2}\right)\left(x_{2}+e_{1}\right), y_{2}=\frac{1}{a}\left(1-x_{1}\right)\left(x_{1}+e_{1}\right)\right\}, \\
& C_{0}^{4 r}=\left\{0<x_{1}<\frac{1-e_{1}}{2}, 0<x_{2}<\frac{1-e_{1}}{2}, y_{1}=\frac{1}{a}\left(1-x_{2}\right)\left(x_{2}+e_{1}\right), y_{2}=\frac{1}{a}\left(1-x_{1}\right)\left(x_{1}+e_{1}\right)\right\} .
\end{aligned}
$$

4.3. Conjecture of the System. In this section, we combine the result of Subsection 4.1 and Subsection 4.2 and propose a conjecture.

Conjecture 1. If $x_{*}>\left(1-e_{1} / 2\right)$ and $\left(x_{*} / x_{*}+e_{1}\right)\left(2 x_{*}+e_{1}-\right.$ $1)+\varepsilon=0$, then the equilibrium $E_{*}\left(x_{*}, y_{*}\right)$ is a Hopf bifurcation point of system (51) and a periodic solution is bifurcated from $E_{*}\left(x_{*}, y_{*}\right)$. Moreover, the relaxation oscillation cycle of the system (51) is unique.

4.4. Numerical Simulation. We show some numerical simulations in this part to verify our theoretical results.
For system (32), we choose the parameter values $\left(a, e_{1}, e_{2}, \varepsilon\right)=(0.805,0.2,0.022,0.01)$, then we get $\left(x_{*}, y_{*}\right)=(0.422,0.444)$. A series of calculations gives us that $\tau_{00} \approx 11.50$. Thus, we choose $\left(\tau_{1}, \tau_{2}\right)=(3,1)$, and then the equilibrium $E_{*}\left(x_{*}, y_{*}\right)$ of system (32) is asymptotically stable (Figure 6). Besides, we choose $\left(\tau_{1}, \tau_{2}\right)=(3,13)$ and get the periodic solutions of system (32) (Figure 7).

For system (51), we choose the parameter values $\left(a, e_{1}, e_{2}, \varepsilon\right)=(1,0.2,0.022,0.01)$. Figure 8 shows the phase portrait and the time series of the relaxation oscillation cycle. 


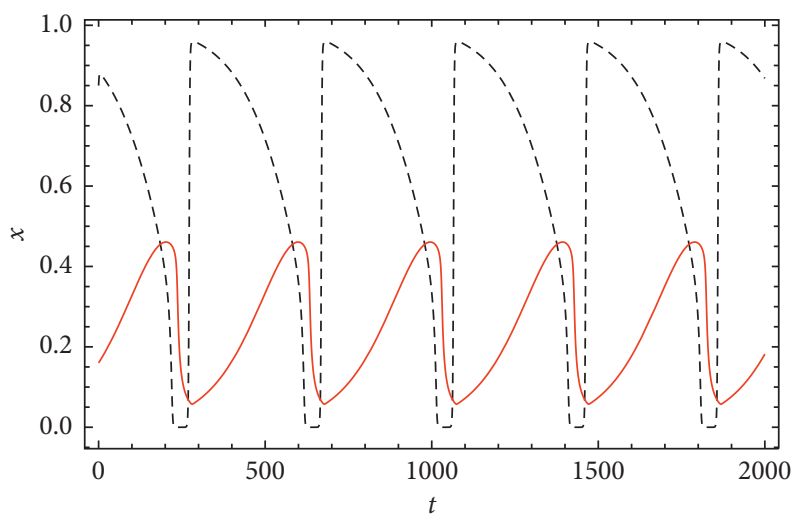

Figure 7: Choose $\left(\tau_{1}, \tau_{2}\right)=(3,13)$ satisfying $\tau_{1}+\tau_{2}=16>\tau_{00}$, and then reach the periodic solutions of system (32) (time series with the black dashed curve (prey) and red full curve (predator)).

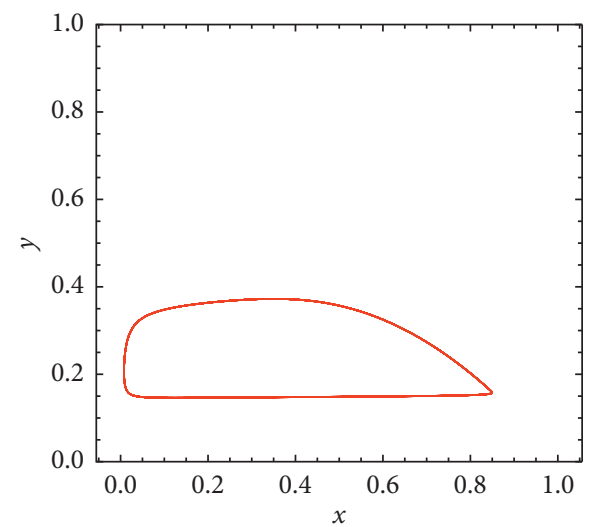

(a)

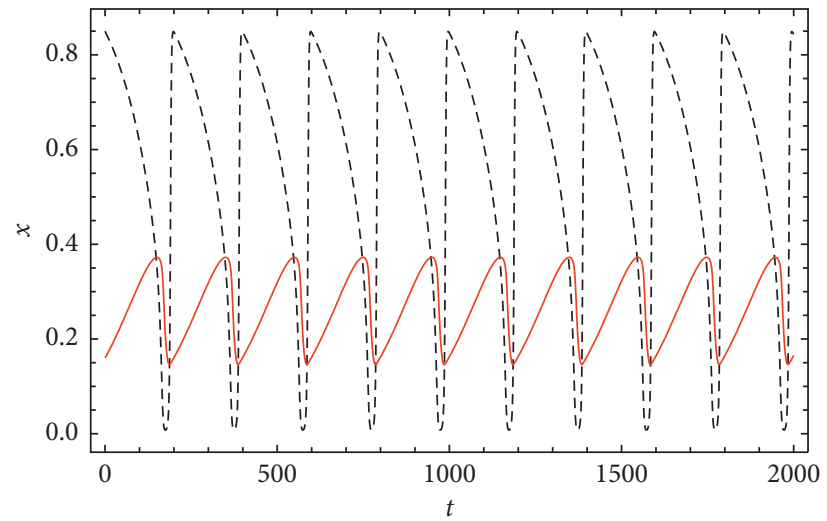

(b)

Figure 8: Numerical simulation of system (51). (a) The orbit is shown in red in the phase space. (b) Time series with the black dashed curve (prey) and red full curve (predator).

\section{Conclusion}

In this paper, we mainly study two kinds of time-delay slow-fast modified Leslie-Gower models. For the first system, through the geometric singular perturbation theory and entry-exit function, we prove the existence and uniqueness of relaxation oscillation cycle. For another system, when $\tau=\tau_{1}+\tau_{2}$ crosses some critical values $\tau_{0 j}$, the Hopf bifurcation occurs. Meanwhile, we put forward a conjecture that the relaxation oscillation cycle of the system is unique when $\tau=0$. Numerical simulation verified our theoretical results and indicated that our method is effective. Our results show that delay affects the stability of the positive equilibrium and produces more complex dynamics than the model without delay. $[1,2]$

\section{Data Availability}

All data, models, and code generated or used during the study are included within the article.

\section{Conflicts of Interest}

The authors declare that they have no conflicts of interest.

\section{Acknowledgments}

The authors gratefully acknowledge the support of the National Natural Science Foundation of China (NNSFC) through grant no.11572288 and the Natural Science Foundation of Zhejiang through grant no.LY20A020003.

\section{References}

[1] M. A. Aziz-Alaoui and M. Daher Okiye, "Boundedness and global stability for a predator-prey model with modified Leslie-Gower and Holling-type II schemes," Applied Mathematics Letters, vol. 16, no. 7, pp. 1069-1075, 2003.

[2] Y. Zhu and K. Wang, "Existence and global attractivity of positive periodic solutions for a predator-prey model with modified Leslie-Gower Holling-type II schemes," Journal of Mathematical Analysis and Applications, vol. 384, no. 2, pp. 400-408, 2011.

[3] N. Fenichel, "Geometric singular perturbation theory for ordinary differential equations," Journal of Differential Equations, vol. 31, no. 1, pp. 53-98, 1979.

[4] C. Kuehn, Multiple Time Scale Dynamics Applied Mathematical Sciences, Springer, Berlin, Germany, 2015. 
[5] G. Hek, "Geometric singular perturbation theory in biological practice," Journal of Mathematical Biology, vol. 60, no. 3, pp. 347-386, 2010.

[6] C. K. R. T. Jones, "Geometric singular perturbation theory," Dynamical Systems, vol. 1609, pp. 44-118, 1995.

[7] W. Liu, "Exchange lemmas for singular perturbation problems with certain turning points," Journal of Differential Equations, vol. 167, no. 1, pp. 134-180, 2000.

[8] W. Liu, "Geometric singular perturbations for multiple turning points: invariant manifolds and exchange lemmas," Journal of Dynamics and Differential Equations, vol. 18, no. 3, pp. 667-691, 2006.

[9] M. Krupa and P. Szmolyan, "Extending geometric singular perturbation theory to nonhyperbolic points---fold and canard points in two dimensions," SIAM Journal on Mathematical Analysis, vol. 33, no. 2, pp. 286-314, 2001.

[10] M. Krupa and P. Szmolyan, "Relaxation oscillation and canard explosion," Journal of Differential Equations, vol. 174, no. 2, pp. 312-368, 2001.

[11] M. Krupa and M. Wechselberger, "Local analysis near a folded saddle-node singularity," Journal of Differential Equations, vol. 248, no. 12, pp. 2841-2888, 2010.

[12] R. Arditi and L. R. Ginzburg, "How species interact: altering the standard view on trophic ecology," Environmental Health Perspectives, vol. 114, no. 11, pp. 142-143, 2012.

[13] S. Schecter and I. Deng's lemma, "Exchange lemmas 1: deng's lemma," Journal of Differential Equations, vol. 245, no. 2, pp. 392-410, 2008.

[14] S. Schecter, "Exchange lemmas 2: general exchange lemma," Journal of Differential Equations, vol. 245, no. 2, pp. 411-441, 2008.

[15] C. Wang and X. Zhang, "Relaxation oscillations in a slow-fast modified Leslie-Gower model," Applied Mathematics Letters, vol. 87, pp. 147-153, 2019.

[16] V. A. Gaiko and C. Vuik, "Global dynamics in the leslie-gower model with the allee Effect," International Journal of Bifurcation and Chaos, vol. 28, no. 12, Article ID 1850151, 2018.

[17] Y. F. Du, B. Niu, and J. J. Wei, "Two delays induce Hopf bifurcation and double Hopf bifurcation in a diffusive LeslieGower predator-prey system," Chaos, vol. 29, Article ID 013101, 2019.

[18] H. M. N. Karl, A. Peter, and D. D. Peter, "Bifurcation of critical sets and relaxation oscillations in singular fast-slow systems," Nonlinearity, vol. 33, pp. 2853-2904, 2020.

[19] C. Wang and X. Zhang, "Stability loss delay and smoothness of the return map in slow-fast systems," SIAM Journal on Applied Dynamical Systems, vol. 17, no. 1, pp. 788-822, 2018.

[20] B. Ambrosio, M. A. Aziz-Alaoui, and R. Yafia, "Canard phenomenon in a slow-fast modified Leslie-Gower model," Mathematical Biosciences, vol. 295, pp. 48-54, 2018.

[21] Y. Xia, Z. Zhang, and Q. Bi, "Relaxation oscillations and the mechanism in a periodically excited vector field with pitchfork-Hopf bifurcation," Nonlinear Dynamics, vol. 101, no. 1, pp. 37-51, 2020.

[22] A. Atabaigi and A. Barati, "Relaxation oscillations and canard explosion in a predator-prey system of Holling and Leslie types," Nonlinear Analysis: Real World Applications, vol. 36, pp. 139-153, 2017.

[23] S. Ai and S. Sadhu, "The entry-exit theorem and relaxation oscillations in slow-fast planar systems," Journal of Differential Equations, vol. 268, no. 11, pp. 7220-7249, 2020. 